[16] Medrinal, C., Prieur, G., Frenoy, É., Robledo Quesada, A., Poncet, A., Bonnevie, T. et. al. (2016). Respiratory weakness after mechanical ventilation is associated with one-year mortality - a prospective study. Critical Care, 20 (1). doi: http://doi.org/ 10.1186/s13054-016-1418-y

[17] DiNino, E., Gartman, E. J., Sethi, J. M., McCool, F. D. (2013). Diaphragm ultrasound as a predictor of successful extubation from mechanical ventilation. Thorax, 69 (5), 431-435. doi: http://doi.org/10.1136/thoraxjnl-2013-204111

[18] Kim, W. Y., Suh, H. J., Hong, S.-B., Koh, Y., Lim, C.-M. (2011). Diaphragm dysfunction assessed by ultrasonography: Influence on weaning from mechanical ventilation. Critical Care Medicine, 39 (12), 2627-2630. doi: http://doi.org/10.1097/ ccm.0b013e3182266408

[19] Carámbula, A., Visca, A., D’Amico, S., Angulo, M. (2019). Evaluación muscular respiratoria y periférica en la Unidad de Cuidados Intensivos. Archivos de Bronconeumología, 55 (5), 258-265. doi: http://doi.org/10.1016/j.arbres.2018.09.002

[20] Kneyber, M. C. J., de Luca, D., Calderini, E., Jarreau, P.-H., Javouhey, E. et. al. (2017). Recommendations for mechanical ventilation of critically ill children from the Paediatric Mechanical Ventilation Consensus Conference (PEMVECC). Intensive Care Medicine, 43 (12), 1764-1780. doi: http://doi.org/10.1007/s00134-017-4920-z

[21] Goligher, E. C., Fan, E., Herridge, M. S., Murray, A., Vorona, S., Brace, D. et. al. (2015). Evolution of Diaphragm Thickness during Mechanical Ventilation. Impact of Inspiratory Effort. American Journal of Respiratory and Critical Care Medicine, 192 (9), 1080-1088. doi: http://doi.org/10.1164/rccm.201503-0620oc

[22] Schepens, T., Verbrugghe, W., Dams, K., Corthouts, B., Parizel, P. M., Jorens, P. G. (2015). The course of diaphragm atrophy in ventilated patients assessed with ultrasound: a longitudinal cohort study. Critical Care, 19 (1). doi: http://doi.org/10.1186/ s13054-015-1141-0

\title{
CYTOKERATIN-18 AS A MARKER OF NON-ALCOHOLIC FATTY LIVER DISEASE IN OBESE ADOLESCENTS
}

\author{
Margaryta Khomenko \\ Department of Adolescent Medicine \\ Kharkiv Medical Academy of Postgraduate Education \\ 58 Amosova str., Kharkiv, Ukraine, 61000 \\ kh.margaryta@gmail.com
}

\begin{abstract}
In parallel with the obesity epidemic in the world, the prevalence of non-alcoholic fatty liver disease among children and adolescents is growing. Current data suggest that insulin resistance is one of the main factors in the pathogenesis of non-alcoholic fatty liver disease, and the content of fragments of caspase-cleaved cytokeratin-18 in the blood serum may be one of the informative indicators of non-alcoholic fatty liver disease progression.

The aim. To determine mechanisms of formation and progression of non-alcoholic fatty liver disease in obese children and adolescents by evaluating the level of cytokeratin-18.

Materials and methods. The study involved 46 adolescents with obesity and non-alcoholic fatty liver disease aged 12 17 years: 19 boys (41.3\%) and 27 girls (58.7\%). Clinical (weight, height, waist and hip circumference), laboratory (glucose, immunoreactive insulin, lipid metabolism, alanine aminotransferase, aspartate aminotransferase, gamma-glutamyl transpeptidase, cytokeratin-18) parameters were studied and instrumental examination (abdominal ultrasound) was performed. To assess insulin resistance the triglyceride-glucose index was calculated.

Results. Depending on the presence of insulin resistance patients were divided into two groups: 21 (45.7\%) of adolescents with insulin resistance and 25 (54.3\%) of adolescents without insulin resistance. Blood tests in patients with insulin resistance revealed significantly higher levels of total cholesterol, triglycerides, very low-density lipoprotein cholesterol, fasting immunoreactive insulin, cytokeratin-18 and gamma-glutamyl transpeptidase. All adolescents were divided into 2 groups depending on the level of cytokeratin-18: patients with cytokeratin-18 $>233 \mathrm{mIU} / \mathrm{ml}$ and $<233 \mathrm{mIU} / \mathrm{ml}(15$ (32.6 \%) and 31 (67.4 \%)
\end{abstract}


respectively). It was found that there were significantly more patients with insulin resistance in the group with the level of cytokeratin-18 $>233 \mathrm{mIU} / \mathrm{ml}$.

Conclusion. In obese adolescents with non-alcoholic fatty liver disease insulin resistance is associated with more pronounced disorders of lipid and carbohydrate metabolism and higher levels of markers that characterize the state of the liver such as cytokeratin-18 and gamma-glutamyl transpeptidase. Adolescents with obesity and non-alcoholic fatty liver disease with a threshold level of cytokeratin-18, which indicates the transformation of steatosis into steatohepatitis, two times more often have present insulin resistance.

Keywords: obesity, non-alcoholic fatty liver disease, triglyceride-glucose index, cytokeratin-18, adolescents.

DOI: $10.21303 / 2504-5679.2020 .001415$

\section{Introduction}

Non-alcoholic fatty liver disease (NAFLD) is the most common cause of chronic liver disease in children and adolescents in the developed world. NAFLD is associated with a number of metabolic risk factors, including obesity, dyslipidemia, insulin resistance, and cardiovascular disease, and is considered a hepatic manifestation of metabolic syndrome [1, 2]. The prevalence of NAFLD among children and adolescents is growing in parallel with the global obesity epidemic. According to the significant research of recent years, $34.2 \%$ of obese children and adolescents suffer from NAFLD, which is 4.5 times more than in the general population [3]. Pathological changes in NAFLD are characterized by stages: vesicular steatosis of the liver can subsequently progress to non-alcoholic steatohepatitis (NASH), fibrosis, cirrhosis, which is the foundation for the development of hepatocellular carcinoma in adults [2]. The "gold standard" for assessing the condition of liver tissue is a biopsy. But due to the fact that the procedure is technically demanding and involves a risk of complications, a number of serum biomarkers are currently being studied to assess pathological changes in the organ.

Cytokeratin-18 (CK-18) is a fragment of intermediate filaments of liver epithelial cells, and apoptosis of hepatocytes is considered one of the main mechanisms of NAFLD development. During apoptosis, activation of caspases occurs, which leads to proteolysis of cellular proteins including CK-18. This process can further cause the activation of stellate cells associated with fibrosis processes [4]. Therefore, the content of fragments of caspase-cleaved cytokeratin-18 in the serum, according to many researchers, may be one of the informative indicators of inflammation and fibrosis in NAFLD.

A key component of obesity and NAFLD is insulin resistance (IR), and although it is currently considered a debatable question about cause-and-effect relationships in this triad, the leading idea is that these are inextricably linked processes [5]. The most accurate tool for assessing the action of insulin is the hyperinsulinemic-euglycemic clamp technique [6]. However, this method is expensive, time-consuming and thus not widely used in clinical practice. Instead, a number of indices have been developed to determine IR, including HOMA-IR, Caro, Matsuda, QUICKI, etc. [7, 8]. Based on the fact that insulin has a significant effect on lipid metabolism, a new tool for calculating IR has recently been proposed - the triglyceride-glucose index [9].

Current data indicate that IR is one of the main factors in the progression of NAFLD from simple steatosis in the NASH $[10,11]$. Thus, today the issue of the influence of insulin resistance on the indicators characterizing the state of the liver in patients with NAFLD in children and adolescents is relevant.

The aim. To determine mechanisms of formation and progression of non-alcoholic fatty liver disease in obese children and adolescents by evaluating the level of cytokeratin - 18 .

\section{Materials and methods}

The study involved 46 adolescents with obesity and NAFLD aged 12-17 years: 19 boys $(41.3 \%)$ and 27 girls $(58.7 \%)$ who were treated at the SI "Institute for Children and Adolescent Health Care at the National Academy of Medical Sciences of Ukraine" in 2018-2019. The diagnosis of obesity was established according to the ICD-10. NAFLD was diagnosed on the basis of an abdominal ultrasound examination. Other causes of liver disease were excluded in all patients. 
Anthropometric examination included measurement of standing height, body weight, waist circumference (WC), hip circumference $(\mathrm{HC})$, calculation of the $\mathrm{WC} / \mathrm{HC}$ ratio, the $\mathrm{WC} /$ height ratio (indicators of abdominal obesity), body mass index (BMI) using the formula: BMI=body weight (kg)/ height $\left(\mathrm{m}^{2}\right)$. The BMI was assessed according to the standards of WHO. The study was conducted in accordance with the requirements of the Helsinki Declaration of the World Medical Association and approved by the local ethics committee of the "Kharkiv Medical Academy of Postgraduate Education" (protocol No. 1, 18.02.2020). All patients signed informed consent for participating in the clinical trial.

The venous blood samples were collected after 10-12 hours of night fasting. The concentrations of serum glucose, immunoreactive insulin (IRI), alanine aminotransferase (ALT), aspartate aminotransferase (AST), gamma-glutamyl transpeptidase (GGTP), total bilirubin, triglycerides (TG), total cholesterol (TC), high-density lipoprotein cholesterol (HDL-C) were measured.

The concentrations of low-density lipoprotein cholesterol (LDL-C) and very low-density lipoprotein cholesterol (VLDL-C) were calculated using formula [12]:

$$
\begin{gathered}
\text { LDL-C }(\mathrm{mmol} / \mathrm{l})=\mathrm{TC}(\mathrm{mmol} / \mathrm{l})-\mathrm{HDL}-\mathrm{C}(\mathrm{mmol} / \mathrm{l})-\mathrm{TG}(\mathrm{mmol} / \mathrm{l}) / 2.2 ; \\
\text { VLDL-C }(\mathrm{mmol} / \mathrm{l})=\mathrm{TG}(\mathrm{mmol} / \mathrm{l}) / 2.2 .
\end{gathered}
$$

Insulin resistance was assessed by calculating the triglyceride-glucose (TyG) index:

$$
\text { TyG index }=\operatorname{Ln}[\text { fasting TG }(\mathrm{mg} / \mathrm{dl}) \times \text { fasting glucose } \mathrm{mg} / \mathrm{dl})] / 2 \text {, }
$$

where $1 \mathrm{mmol} / \mathrm{l}$ of TG equals $88.57 \mathrm{mg} / \mathrm{dl}, 1 \mathrm{mmol} / 1$ of glucose equals $18 \mathrm{mg} / \mathrm{dL}$.

According to the method, the value of the index $\geq 4.75$ for girls and $\geq 4.70$ for boys indicates the presence of IR [9].

The level of cytokeratin-18 was determined by using an ELISA kit (CUSABIO, USA). U1trasound examination was performed on an empty stomach using an «ULTIMA SM-50» with a 3.5-5 MHz convex transducer and a 7.5-10 MHz linear transducer.

Statistical processing of material was performed using Microsoft Excel, Statistica 6.0. The data were expressed as mean \pm SE. The distribution of the data was evaluated with the Kolmogorov-Smirnov test. Differences between groups were tested using Student's t-test and chi-squared test $\left(\chi^{2}\right)$. A $p$-value $<0.05$ was considered statistically significant.

\section{Results}

Depending on the presence of IR, patients were divided into two groups: 21 (45.7\%) of adolescents with IR - I group (IR“+”) and 25 (54.3\%) of adolescents without IR - II group (IR“_"). The study revealed that $37(80.4 \%)$ of patients had increased appetite, $26(56.5 \%)$ - abdominal pain, $19(41.3 \%)$ - belching, 17 (36.9\%) - heartburn, 15 (32.6\%) - nausea, 14 (30.4\%) - flatulence, $11(23.9 \%)$ - constipation, and $8(17.4 \%)$ had loose stools. Objective examination of patients revealed pain in the right hypochondrium in $21(45.6 \%)$ of adolescents, in the epigastrium in 16 (34.7\%), liver enlargement was noted in $44(95.6 \%)$ patients. The frequency of complaints did not differ depending on the presence of IR.

When determining anthropometric parameters significantly higher in patients with IR were BMI $\left(32.91 \pm 0.4 \mathrm{~kg} / \mathrm{m}^{2}\right.$ vs $\left.30.09 \pm 0.62 \mathrm{~kg} / \mathrm{m}^{2}, \mathrm{p} \leq 0.01\right)$ and the $\mathrm{WC} /$ height ratio $(0.58 \pm 0.01 \mathrm{vs}$ $0.55 \pm 0.03, \mathrm{p} \leq 0.05)$. The characteristic of laboratory parameters of the examined adolescents is presented in Table 1.

Among the laboratory parameters significantly higher in patients of group I were the levels of TC $(p \leq 0.01)$, TG $(p \leq 0.01)$, VLDL-C $(p \leq 0.01)$, fasting IRI $(p \leq 0.05)$, GGTP $(p \leq 0.05)$, and the levels of glucose, LDL-C, HDL-C did not differ. Significantly higher level of CK-18 $(p \leq 0.05)$ was observed in the group of adolescents with IR (Fig. 1).

In the study of Feldstein A.E. et al. the level of cytokeratin-18 in children with biopsy proven NAFLD was analyzed [13]. It was concluded that the value of $233 \mathrm{mIU} / \mathrm{ml}$ has the highest 
sensitivity and specificity (85.0 \% and $86.9 \%$ respectively) for the establishment of NASH. Using these data, all adolescents in our study were divided into 2 groups depending on the level of cytokeratin-18: group I - 15 patients with cytokeratin-18 $>233 \mathrm{mIU} / \mathrm{ml}$ (32.6\%), group II 31 patients with cytokeratin- $18<233 \mathrm{mIU} / \mathrm{ml}(67.4 \%)$. It was found that there were significantly more patients with insulin resistance in group I than in the group of adolescents with the level of cytokeratin-18 $<233 \mathrm{mIU} / \mathrm{ml}(10(66.7 \%)$ and $11(35.5 \%)$, respectively, $\mathrm{p} \leq 0.05)$.

Table 1

The characteristic of laboratory parameters of the examined adolescents depending on the presence of insulin resistance (mean $\pm \mathrm{SE})$

\begin{tabular}{ccc}
\hline Parameters & I group, IR“+” (n=21) & II group, IR“_”(n=25) \\
\hline Total bilirubin $(\mu \mathrm{mol} / \mathrm{l})$ & $15.58 \pm 1.16$ & $16.78 \pm 0.97$ \\
Total cholesterol $(\mathrm{mmol} / \mathrm{l})$ & $5.58 \pm 0.21^{* *}$ & $4.92 \pm 0.14$ \\
Triglycerides $(\mathrm{mmol} / \mathrm{l})$ & $1.89 \pm 0.09^{* *}$ & $1.07 \pm 0.04$ \\
LDL-C $(\mathrm{mmol} / \mathrm{l})$ & $3.19 \pm 0.19$ & $2.760 .14 \pm$ \\
VLDL-C $(\mathrm{mmol} / \mathrm{l})$ & $0.84 \pm 0.04^{* *}$ & $0.48 \pm 0.02$ \\
HDL-C $(\mathrm{mmol} / \mathrm{l})$ & $1.54 \pm 0.07$ & $1.67 \pm 0.08$ \\
Glucose $(\mathrm{mmol} / \mathrm{l})$ & $4.91 \pm 0.11$ & $4.78 \pm 0.07$ \\
IRI $(\mu \mathrm{IU} / \mathrm{ml})$ & $29.3 \pm 2.81^{*}$ & $20.61 \pm 2.33$ \\
AST $(\mathrm{U} / \mathrm{L})$ & $27.61 \pm 2.58$ & $26.75 \pm 1.95$ \\
ALT $(\mathrm{U} / \mathrm{L})$ & $28.01 \pm 2.97$ & $27.87 \pm 2.25$ \\
GGTP $(\mathrm{U} / \mathrm{L})$ & $23.85 \pm 1.31^{*}$ & $21.17 \pm 1.35$ \\
CK-18 $(\mathrm{mlU} / \mathrm{ml})$ & $226.85 \pm 19.17^{*}$ & $181.71 \pm 11.43$
\end{tabular}

Note: AST - aspartate aminotransferase; ALT - alanine aminotransferase; GGTP - gamma-glutamyl transpeptidase; VLDL-C very low-density lipoprotein cholesterol; $L D L$-C -low-density lipoprotein cholesterol; $H D L-C$ - high-density lipoprotein cholesterol; IRI-immunoreactive insulin; CK-18 - cytokeratin-18. Statistically significant difference: ${ }^{*}-p \leq 0.05 ; * *-p \leq 0.01$

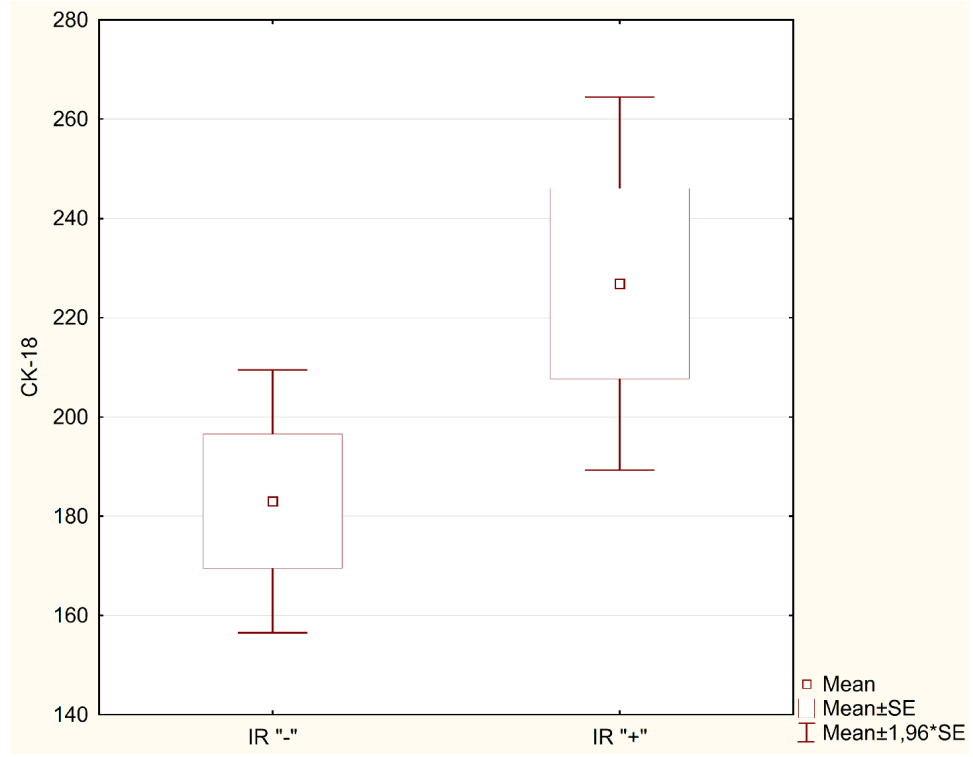

Fig. 1. The level of cytokeratin-18 depending on the presence of insulin resistance in obese adolescents with NAFLD: IR "+" - the group of adolescents with insulin resistance, IR “-” - the group of adolescents without insulin resistance 


\section{Discussion}

Currently, an active study of the relationship between IR and NAFLD is being conducted. The triglyceride-glucose index is proposed as a new alternative marker for determining IR. The reliability of the tool is confirmed by studies that have shown its compliance with the results obtained by the method of hyperinsulinemic euglycemic clamp and the calculation of other known indices for determining IR in both adults and pediatric population [14-16].

Schwimmer J. et al. were among the first who investigated the association of IR and NAFLD in obese children and adolescents. The study demonstrated significantly higher levels of the insulin resistance index (HOMA-IR) in children with NAFLD compared to the values of healthy peers [17]. Since then, the relationship between NAFLD and IR has also been described in other studies involving children and adolescents.

In present study, such anthropometric parameters as BMI and the $\mathrm{WC} /$ height ratio, which characterize abdominal obesity associated to NAFLD, were linked to higher levels of TyG. This is consistent with data from a study by Calcaterra V. et al, where a positive association of TyG index with BMI and the WC/height ratio in obese children was observed [18]. As in the work of Locateli J. C. et al. [19] present study demonstrated the deterioration of carbohydrate and lipid metabolism in the presence of IR, based on calculating TyG index in obese children and adolescents with NAFLD.

The discovery of new biomarkers that characterize the state of the liver contributes to a better understanding of the mechanisms underlying the relationship between IR, obesity and NAFLD. According to data of many researchers, the level of fragments of caspase-cleaved CK-18 in the blood serum may be one of the informative indicators of the progression of NAFLD [20, 21]. Thus, the determination of the level of CK-18 was proposed as a marker for predicting the presence of NASH in the study of Feldstein A. et al. [13] and fibrosis in the works of Fitzpatrick E. et al., Lebensztejn D.M. et al. [22, 23] in children and adolescents with NAFLD. Interesting was the study of Vuppalanchi et al., which revealed a significant decrease in the level of CK-18 with an improvement in the dynamics of NAFLD in children after treatment [24].

It was noted in the report of the ESPGHAN Hepatology Committee that high GGTP levels are associated with fibrotic changes in the liver [25]. In the present study, higher levels of CK-18 and GGTP were found in adolescents from the group with existing IR, which indicates the influence of IR on the progression of NAFLD.

The limitation of the study was the lack of additional verification of the diagnosis of NAFLD by ultrasound shear wave elastography.

Prospects for further research. The search and study of potential biomarkers that characterize the condition of the liver will help improve the diagnosis and prognosis of NAFLD in obese children and adolescents.

\section{Conclusions}

1. In adolescents with obesity and NAFLD, atherogenic changes in the lipid spectrum are detected, which are manifested by increased levels of total cholesterol, triglycerides and very low-density lipoproteins. These indicators are significantly higher in patients with present insulin resistance.

2. Adolescents with obesity and NAFLD have higher activity of gamma-glutamyl transpeptidase on the background of insulin resistance, which may indicate the progression of steatosis towards steatohepatitis.

3. Higher level of cytokeratin-18 is revealed in adolescents with obesity and NAFLD in the presence of insulin resistance.

4. Adolescents with obesity and NAFLD with a threshold level of cytokeratin-18, which indicates the transformation of steatosis into steatohepatitis, two times more often have present insulin resistance.

Thus, the presented study confirms the possibility of fibrotic changes and progression of the inflammatory process in the liver in obese adolescents with NAFLD, which are more likely to occur on the background of insulin resistance. 


\section{Conflict of interest}

The authors declare that they have no conflicts of interest.

\section{References}

[1] Wittcopp, C., Conroy, R. (2016). Metabolic Syndrome in Children and Adolescents. Pediatrics in Review, 37 (5), $193-202$. doi: http://doi.org/10.1542/pir.2014-0095

[2] Bush, H., Golabi, P., Younossi, Z. M. (2017). Pediatric Non-Alcoholic Fatty Liver Disease. Children, 4 (6), 48. doi: http:// doi.org/10.3390/children4060048

[3] Anderson, E. L., Howe, L. D., Jones, H. E., Higgins, J. P. T., Lawlor, D. A., Fraser, A. (2015). The Prevalence of Non-Alcoholic Fatty Liver Disease in Children and Adolescents: A Systematic Review and Meta-Analysis. PLOS ONE, 10 (10), e0140908. doi: http://doi.org/10.1371/journal.pone.0140908

[4] Mandelia, C., Kabbany, M. N., Conjeevaram Selvakumar, P. K., Alkhouri, N. (2018). The search for noninvasive methods to identify liver fibrosis in children with nonalcoholic fatty liver disease. Biomarkers in Medicine, 12 (3), 265-273. doi: http:// doi.org/10.2217/bmm-2017-0038

[5] Levy-Marchal, C., Arslanian, S., Cutfield, W., Sinaiko, A., Druet, C., Marcovecchio, M. L., Chiarelli, F. (2010). Insulin Resistance in Children: Consensus, Perspective, and Future Directions. The Journal of Clinical Endocrinology \& Metabolism, 95 (12), 5189-5198. doi: http://doi.org/10.1210/jc.2010-1047

[6] Tagi, V. M., Giannini, C., Chiarelli, F. (2019). Insulin Resistance in Children. Frontiers in Endocrinology, 10. doi: http:// doi.org/10.3389/fendo.2019.00342

[7] Singh, B. (2010). Surrogate markers of insulin resistance: A review. World Journal of Diabetes, 1 (2), 36. doi: http:// doi.org/10.4239/wjd.v1.i2.36

[8] Frithioff-Bøjsøe, C., Lund, M. A. V., Lausten-Thomsen, U., Hedley, P. L., Pedersen, O., Christiansen, M. et. al. (2019). Leptin, adiponectin, and their ratio as markers of insulin resistance and cardiometabolic risk in childhood obesity. Pediatric Diabetes, 21 (2), 194-202. doi: http://doi.org/10.1111/pedi.12964

[9] Rodríguez-Morán, M., Simental-Mendía, L., Guerrero-Romero, F. (2017). The triglyceride and glucose index is useful for recognising insulin resistance in children. Acta Paediatrica, 106 (6), 979-983. doi: http://doi.org/10.1111/apa.13789

[10] Alam, S., Mustafa, G., Alam, M., Ahmad, N. (2016). Insulin resistance in development and progression of nonalcoholic fatty liver disease. World Journal of Gastrointestinal Pathophysiology, 7 (2), 211. doi: http://doi.org/10.4291/wjgp.v7.i2.211

[11] Strashok, L. A., Buznytska, O. V. (2019). Criteria for the progression of steatohepatosis in obese adolescents with signs of metabolic syndrome. CHILD`S HEALTH, 14 (6), 366-373. doi: http://doi.org/10.22141/2224-0551.14.6.2019.179244

[12] Friedewald, W. T., Levy, R. I., Fredrickson, D. S. (1972). Estimation of the Concentration of Low-Density Lipoprotein Cholesterol in Plasma, Without Use of the Preparative Ultracentrifuge. Clinical Chemistry, 18 (6), 499-502. doi: http://doi.org/ 10.1093/clinchem/18.6.499

[13] Feldstein, A. E., Alkhouri, N., De Vito, R., Alisi, A., Lopez, R., Nobili, V. (2013). Serum Cytokeratin-18 Fragment Levels Are Useful Biomarkers for Nonalcoholic Steatohepatitis in Children. American Journal of Gastroenterology, 108 (9), $1526-1531$. doi: http://doi.org/10.1038/ajg.2013.168

[14] Khan, S. H., Sobia, F., Niazi, N. K., Manzoor, S. M., Fazal, N., Ahmad, F. (2018). Metabolic clustering of risk factors: evaluation of Triglyceride-glucose index (TyG index) for evaluation of insulin resistance. Diabetology \& Metabolic Syndrome, 10 (1). doi: http://doi.org/10.1186/s13098-018-0376-8

[15] Mohd Nor, N. S., Lee, S., Bacha, F., Tfayli, H., Arslanian, S. (2015). Triglyceride glucose index as a surrogate measure of insulin sensitivity in obese adolescents with normoglycemia, prediabetes, and type 2 diabetes mellitus: comparison with the hyperinsulinemic-euglycemic clamp. Pediatric Diabetes, 17 (6), 458-465. doi: http://doi.org/10.1111/pedi.12303

[16] Fiorentino, T. V., Marini, M. A., Succurro, E., Andreozzi, F., Sesti, G. (2019). Relationships of surrogate indexes of insulin resistance with insulin sensitivity assessed by euglycemic hyperinsulinemic clamp and subclinical vascular damage. BMJ Open Diabetes Research \& Care, 7 (1), e000911. doi: http://doi.org/10.1136/bmjdrc-2019-000911

[17] Schwimmer, J. B., Deutsch, R., Rauch, J. B., Behling, C., Newbury, R., Lavine, J. E. (2003). Obesity, insulin resistance, and other clinicopathological correlates of pediatric nonalcoholic fatty liver disease. The Journal of Pediatrics, 143 (4), 500-505. doi: http://doi.org/10.1067/s0022-3476(03)00325-1

[18] Calcaterra, V., Montalbano, C., de Silvestri, A., Pelizzo, G., Regalbuto, C., Paganelli, V. et. al. (2019). Triglyceride Glucose Index as a Surrogate Measure of Insulin Sensitivity in a Caucasian Pediatric Population. Journal of Clinical Research in Pediatric Endocrinology. doi: http://doi.org/10.4274/jcrpe.galenos.2019.2019.0024

[19] Locateli, J. C., Lopes, W. A., Simões, C. F., de Oliveira, G. H., Oltramari, K., Bim, R. H. et. al. (2019). Triglyceride/ glucose index is a reliable alternative marker for insulin resistance in South American overweight and obese children 
and adolescents. Journal of Pediatric Endocrinology and Metabolism, 32 (10), 1163-1170. doi: http://oi.org/10.1515/ jpem-2019-0037

[20] Neuman, M. G., Cohen, L. B., Nanau, R. M. (2014). Biomarkers in Nonalcoholic Fatty Liver Disease. Canadian Journal of Gastroenterology and Hepatology, 28 (11), 607-618. doi: http://doi.org/10.1155/2014/757929

[21] He, L., Deng, L., Zhang, Q., Guo, J., Zhou, J., Song, W., Yuan, F. (2017). Diagnostic Value of CK-18, FGF-21, and Related Biomarker Panel in Nonalcoholic Fatty Liver Disease: A Systematic Review and Meta-Analysis. BioMed Research International, 2017, 1-12. doi: http://doi.org/10.1155/2017/9729107

[22] Fitzpatrick, E., Mitry, R. R., Quaglia, A., Hussain, M. J., deBruyne, R., Dhawan, A. (2010). Serum Levels of CK18 M30 and Leptin Are Useful Predictors of Steatohepatitis and Fibrosis in Paediatric NAFLD. Journal of Pediatric Gastroenterology and Nutrition, 51 (4), 500-506. doi: http://doi.org/10.1097/mpg.0b013e3181e376be

[23] Lebensztejn, D. M., Wierzbicka, A., Socha, P., Pronicki, M., Skiba, E., Werpachowska, I., Kaczmarski, M. (2011). Cytokeratin-18 and hyaluronic acid levels predict liver fibrosis in children with non-alcoholic fatty liver disease. Acta Biochimica Polonica, 58 (4). doi: http://doi.org/10.18388/abp.2011_2225

[24] Vuppalanchi, R., Jain, A. K., Deppe, R., Yates, K., Comerford, M., Masuoka, H. C. et. al. (2014). Relationship Between Changes in Serum Levels of Keratin 18 and Changes in Liver Histology in Children and Adults With Nonalcoholic Fatty Liver Disease. Clinical Gastroenterology and Hepatology, 12 (12), 2121-2130.e2. doi: http://oi.org/10.1016/ j.cgh.2014.05.010

[25] Vajro, P., Lenta, S., Socha, P., Dhawan, A., McKiernan, P., Baumann, U. et. al. (2012). Diagnosis of Nonalcoholic Fatty Liver Disease in Children and Adolescents. Journal of Pediatric Gastroenterology and Nutrition, 54 (5), 700-713. doi: http:// doi.org/10.1097/mpg.0b013e318252a13f

\title{
COMPARATIVE ASPECTS OF PLACENTAL DYSFUNCTION IN WOMEN USING DIFFERENT METHODS OF CONTRACEPTION IN ANAMNESIS
}

\author{
Oleksandra Lubkovska \\ Department of Obstetrics, Gynecology and Perinatology \\ Ukrainian State Institute of Reproductology \\ Shupyk National Medical Academy of Postgraduate Education \\ 9 Dorohozhytska str., Kyiv, Ukraine, 04112
}

\begin{abstract}
The aim. To reduce the incidence of perinatal pathology in women who have used various methods of contraception, based on the study of the functional state of the fetoplacental complex (FPS), as well as improving diagnostic measures and tactics of pregnancy.

Materials and methods. 140 pregnant women were studied with their division into control and 3 test groups depending on the method of contraception in the anamnesis, using clinical, laboratory and instrumental research methods during pregnancy and childbirth.

Results. The study found that women with a history of intrauterine contraception (IUC), in contrast to combined oral contraception (COC) and combined oral contraception containing folate $(\mathrm{COC}+\mathrm{F})$, had a higher incidence of complications during pregnancy and childbirth, as well as fewer newborns with a satisfactory condition at birth. Assessment of folic acid levels at 6-8 weeks of gestation showed significantly better results among women with a history of COC+F, compared with IUC and COC. In the group of women with IUC in the anamnesis, significantly worse mean endocrinological values prevailed, and there was also a greater number of pregnant women with disorders of fetal-placental blood flow. More pronounced dystrophic changes in the placentas of women in this group were pathomorphologically confirmed.
\end{abstract}

